\title{
Discovery of optically faint obscured quasars with Virtual Observatory tools
}

\author{
P. Padovani ${ }^{1}$, M. G. Allen ${ }^{2}$, P. Rosati ${ }^{3}$, and N. A. Walton ${ }^{4}$ \\ 1 ST-ECF, European Southern Observatory, Karl-Schwarzschild-Str. 2, 85748 Garching bei München, Germany \\ e-mail: Paolo.Padovani@eso.org \\ 2 Centre de Données astronomiques de Strasbourg (UMR 7550), 11 rue de 1'Université, 67000 Strasbourg, France \\ e-mail: allen@astro.u-strasbg.fr \\ 3 European Southern Observatory, Karl-Schwarzschild-Str. 2, 85748 Garching bei München, Germany \\ e-mail: Piero.Rosati@eso.org \\ ${ }^{4}$ Institute of Astronomy, Madingley Road, Cambridge CB3 OHA, UK \\ e-mail: naw@ast.cam.ac.uk
}

Received 23 April 2004 / Accepted 2 June 2004

\begin{abstract}
We use Virtual Observatory (VO) tools to identify optically faint, obscured (i.e., type 2) active galactic nuclei (AGN) in the two Great Observatories Origins Deep Survey (GOODS) fields. By employing publicly available X-ray and optical data and catalogues we discover 68 type 2 AGN candidates. The X-ray powers of these sources are estimated by using a previously known correlation between X-ray luminosity and X-ray-to-optical flux ratio. Thirty-one of our candidates have high estimated powers $\left(L_{\mathrm{x}}>10^{44} \mathrm{erg} / \mathrm{s}\right)$ and therefore qualify as optically obscured quasars, the so-called "QSO 2". Based on the derived $\mathrm{X}$-ray powers, our candidates are likely to be at relatively high redshifts, $z \sim 3$, with the QSO 2 at $z \sim 4$. By going $\sim 3$ mag fainter than previously known type 2 AGN in the two GOODS fields we are sampling a region of redshift - power space which was previously unreachable with classical methods. Our method brings to 40 the number of QSO 2 in the GOODS fields, an improvement of a factor $\sim 4$ when compared to the only 9 such sources previously known. We derive a QSO 2 surface density down to $10^{-15} \mathrm{erg} \mathrm{cm}^{-2} \mathrm{~s}^{-1}$ in the $0.5-8 \mathrm{keV}$ band of $\gtrsim 330 \mathrm{deg}^{-2}, \sim 30 \%$ of which is made up of previously known sources. This is larger than current estimates and some predictions and suggests that the surface density of QSO 2 at faint flux limits has been underestimated. This work demonstrates that VO tools are mature enough to produce cutting-edge science results by exploiting astronomical data beyond "classical" identification limits $(R \lesssim 25)$ with interoperable tools for statistical identification of sources using multiwavelength information.
\end{abstract}

Key words. astronomical data bases: miscellaneous - methods: statistical - galaxies: quasars: general - X-rays: galaxies

\section{Introduction}

The unified model for active galactic nuclei (AGN) is largely accepted (e.g., Urry \& Padovani 1995; see also the very recent results by Jaffe et al. 2004). The apparent disparate properties and nomenclature of active galaxies can be explained by the physics of black hole, accretion disk, jet, and obscuring torus convolved with the geometry of the viewing angle. Type 1 sources are those in which we have an unimpeded view of the central regions and therefore exhibit the straight physics of AGN with no absorption. Type 2 objects arise when the view is obscured by the torus. While many examples of local, and therefore relatively low-power, type 2 AGN are known (the Seyfert 2s), it has been debated if their high-power counterparts, that is optically obscured, radio-quiet type 2 QSO, exist. Indeed, until very recently, very few, if any, examples of this class were known. Apart from their importance for
AGN models, type 2 sources are expected to make a significant fraction of the X-ray background (see, e.g., Comastri et al. 2001) and are therefore also cosmologically very relevant. These sources are heavily reddened and therefore fall through the "standard" (optical) methods of quasar selection. The hard X-rays, however, are thought to be able to penetrate the torus. Type 2 QSO, therefore, should have narrow, if any, permitted lines (and might look like normal galaxies in the optical/UV band), powerful hard X-ray emission, and, in some cases, a high equivalent width Fe K line (e.g., Norman et al. 2002).

In this paper we use Virtual Observatory (VO) tools to identify 68 type 2 AGN candidates in the two Great Observatories Origins Deep Survey (GOODS) fields (Giavalisco et al. 2004a), $\sim 1 / 2$ of which qualify as QSO 2 candidates. Based on the properties of already known sources, we expect the large majority of these to be obscured quasars whose identification is only possible through their X-ray emission. 
VO initiatives are now at a stage where prototype tools can be utilised to produce scientific results. Real gains have been made in the areas of accessing and describing remote data sets, manipulating image and catalogue data, and performing remote calculations in a fashion similar to grid computing. These prototype tools are enabled by the VO infrastructure and interoperability standards that are being developed cooperatively by all the VO projects under the auspices of the IVOA ${ }^{1}$. VO software is expected to mature significantly over the next $1-2$ years as the VO projects progress from demonstrations to building robust systems. In this paper we have taken advantage of the first interoperability gains to produce the "first science" for the VO.

In Sect. 2 we present the data we have used, Sect. 3 discusses the tools we employed, while Sect. 4 describes our method. Section 5 presents our results, which are discussed in Sect. 6, while Sect. 7 summarizes our conclusions. Throughout this paper spectral indices are written $S_{v} \propto v^{-\alpha}$ and we adopt a cosmological model with $H_{0}=70 \mathrm{~km} \mathrm{~s}^{-1} \mathrm{Mpc}^{-1}, \Omega_{\mathrm{M}}=0.3$, and $\Omega_{\Lambda}=0.7$.

\section{The data}

We use for our purposes the two GOODS fields (Giavalisco et al. 2004a), namely the Hubble Deep Field-North (HDF-N) and the Chandra Deep Field-South (CDF-S). Since GOODS includes some of the deepest observations from space- and ground-based facilities, these are the most data-rich, deep survey areas on the sky. The GOODS field centres (J2000.0) are $12^{\mathrm{h}} 36^{\mathrm{m}} 55^{\mathrm{s}},+62^{\circ} 14^{\prime} 15^{\prime \prime}$ for the HDF-N and $3^{\mathrm{h}} 32^{\mathrm{m}} 30^{\mathrm{s}}$, $-27^{\circ} 48^{\prime} 20^{\prime \prime}$ for the CDF-S. Each field provides an area of approximately $10^{\prime} \times 16^{\prime}$.

Deep X-ray (Chandra) catalogues are available for a larger region around both fields (Alexander et al. 2003; Giacconi et al. 2002). For consistency, we use the catalogues produced by Alexander et al. (2003) for both the $2 \mathrm{Ms}$ HDF-N and $1 \mathrm{Ms}$ CDF-S data. These include 503 (HDF-N) and 326 (CDF-S) objects respectively, for a total of 829 sources. The data cover the $0.5-8.0 \mathrm{keV}$ band and the catalogues provide counts and fluxes in various sub-bands. Note that, due to the twice as long exposure time, the HDF-N reaches fainter X-ray fluxes and includes a (54\%) larger number of sources.

In the optical we use the publicly available GOODS Hubble Space Telescope (HST) Advanced Camera for Surveys (ACS) data (proposal ID 9583). The observations consist of imaging in the $F 435 \mathrm{~W}, F 606 \mathrm{~W}, F 775 \mathrm{~W}$, and $F 850 L P$ passbands, hereafter referred to as $B, V, i$, and $z$, respectively. We use here version v1.0 of the reduced, calibrated, stacked, and mosaiced images and catalogues as made available by the GOODS team ${ }^{2}$.

Finally, identifications and redshifts through optical spectroscopy are available from Barger et al. (2003) and Szokoly et al. (2004) for the HDF-N and CDF-S respectively. We note that $\sim 56 \%$ of the X-ray sources in the GOODS fields have spectroscopic redshift determinations. These sources are necessarily relatively bright, with $\langle I\rangle \sim 21$ (HDF-N; only $3 \%$

\footnotetext{
${ }^{1}$ http://www.ivoa.net

2 http://www.stsci.edu/science/goods/
}

fainter than 24th mag) and $\langle R\rangle \sim 22$ (CDF-S; only 5\% fainter than 25th mag).

\section{Virtual Observatory tools}

Astronomy is at a turning point. Major breakthroughs in telescope, detector, and computer technology allow astronomical surveys to produce massive amounts of images, spectra, and catalogues. These datasets cover the sky at all wavelengths from $\gamma$ - and X-rays, optical, infrared, through to radio. The VO is an international, community-based initiative, to allow global electronic access to available astronomical data, both space- and ground-based. The VO aims also to enable data analysis techniques through a coordinating entity that will provide common standards, wide-network bandwidth, and stateof-the-art analysis tools.

The Astrophysical Virtual Observatory Project $(\mathrm{AVO})^{3}$ is conducting a research and demonstration programme on the scientific requirements and technologies necessary to build a VO for European astronomy. The AVO has been jointly funded by the European Commission (under FP5 - Fifth Framework Programme) with six European organisations participating in a three year Phase-A work programme.

The AVO project is driven by its strategy of regular scientific demonstrations of VO technology. For this purpose an "AVO prototype" has been built. The prototype consists of a suite of interoperable software, plus a set of conventions or standards for accessing remote data, and for launching remote calculations. The main component of the software is based on the CDS Aladin visualisation interface (Bonnarel et al. 2000). This prototype VO portal (v. 1.003- $\beta$ ) allows efficient interactive manipulation of image and catalogue data, and provides access to remote data archives and image servers via the GLU registry of services ${ }^{4}$.

The Aladin image server is an example of such a VO service. It describes the images stored in the Aladin database using a data model (Images Distribuées Hétérogènes pour l'Astronomie; IDHA $^{5}$ ), and provides image cutouts on request. In this paper we make heavy use of cutouts of the GOODS data available via this service. The prototype is also interoperable with the other long standing CDS Vizier and SIMBAD services (Ochsenbein et al. 2000), and significant interoperability gains are achieved by use of the VOTable ${ }^{6}$ format for astronomical tables.

The prototype includes a catalogue cross matching service. This service allows positional cross matching of two catalogues to find the best matched source, all matching sources, or sources not matching within a given threshold radius (in arcseconds). These three modes, plus the ability to compare directly with the images from which the catalogues were generated, make for an extremely efficient tool for which to perform cross matches and check for multiple, or aberrant matches.

\footnotetext{
http://www. euro-vo.org/

${ }^{4}$ http://simbad.u-strasbg.fr/glu/glu.htx

5 http://cdsweb.u-strasbg.fr/idha.html

${ }^{6}$ http://cdsweb.u-strasbg.fr/doc/VOTable
} 
In addition to the AVO software, we have also made intensive use of the Starlink topcat $\operatorname{tool}^{7}$, and the VOIndia VOPlot plugin ${ }^{8}$ to the prototype.

\section{The method}

The two key physical properties that we use to identify type 2 AGN candidates are that they be obscured, and that they have sufficiently high power to be classed as an AGN and not a starburst. To find these candidates, we use a relatively simple method based on the X-ray and optical fluxes.

Optical data alone are not sufficient for this purpose because at the redshifts that we expect to find these sources the nuclear, AGN emission can be diluted by the host galaxy. Indeed, Moran et al. (2002) have shown that $\sim 60 \%$ of local Seyfert 2 galaxies would be classified as normal galaxies if no decomposition of their optical emission were available. However, AGN reveal themselves by their hard X-ray emission and power. Ideally, to find type 2 AGN, one would need X-ray spectra to select sources with flat spectral indices, indicative of absorption. The typical count rates of the $\mathrm{X}$-ray sources in the GOODS fields are however relatively low for detailed spectral fitting and therefore we select sources based on their X-ray hardness ratio, a measure of the fraction of hard photons relative to soft photons.

To estimate the X-ray power for candidate type 2 sources we use the correlation described by Fiore et al. (2003) between the $f(2-10 \mathrm{keV}) / f(R)$ ratio and the hard X-ray power (see their Fig. 5). The basis of this correlation is the fact that the $f(2-10 \mathrm{keV}) / f(R)$ ratio is roughly equivalent to the ratio between the nuclear X-ray power and the host galaxy $R$ band luminosity. Since the host galaxy $R$ band luminosities (unlike the X-ray power) show only a modest amount of scatter, this flux ratio is a good indicator of $\mathrm{X}$-ray power.

Each of the steps in our method, including our technique for identifying the optical counterparts of the X-ray sources, are described in detail below.

\subsection{Selecting absorbed sources}

The Alexander et al. (2003) catalogues provide counts in various X-ray bands. We define the hardness ratio $H R=(H-$ $S) /(H+S)$, where $H$ is the hard X-ray counts $(2.0-8.0 \mathrm{keV})$ and $S$ is the soft X-ray counts $(0.5-2.0 \mathrm{keV})$. Following Szokoly et al. (2004) when $H$ is an upper limit we set $H R=$ -1 , while when $S$ is an upper limit we set $H R=1$. The 14 sources with upper limits in both $H$ and $S$ bands were excluded. Szokoly et al. (2004) have shown that absorbed, type 2 AGN are characterized by $H R \geq-0.2$. We adopt this criterion and identify those sources which have $H R \geq-0.2$ as absorbed sources. We find 294 (CDF-S: 104, HDF-N: 190) such absorbed sources which represent $35_{-2}^{+3} \%$ ( $1 \sigma$ Poisson errors are from Gehrels 1986) of the X-ray sources in the Alexander catalogues. The hardness ratio distribution is shown in Fig. 1. We note that for an $\alpha_{\mathrm{x}}=0.8$ the flux in the $2-8 \mathrm{keV}$ band

\footnotetext{
${ }^{7}$ http://www. starlink.ac.uk/topcat/

${ }^{8}$ http://vo.iucaa.ernet.in/ voi/voplot.htm
}

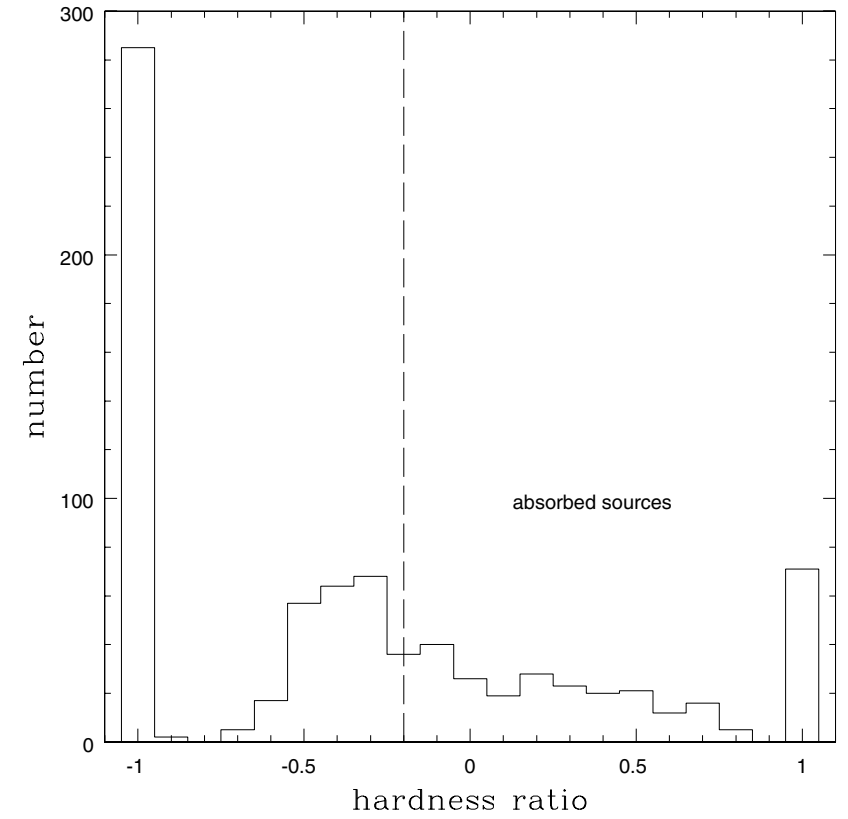

Fig. 1. The distribution of hardness ratios in the Alexander et al. (2003) catalogues. We define as absorbed sources to the right of the dashed line, that is having hardness ratio $H R \geq-0.2$.

used by Alexander et al. (2003) is only $\sim 15 \%$ smaller than that in the commonly used $2-10 \mathrm{keV}$ band. We also point out that increasing redshift makes the sources softer (e.g., at $z=3$ the rest-frame $2-8 \mathrm{keV}$ band shifts to $0.5-2 \mathrm{keV}$ ) so our selection criterion will mistakenly discard some high- $z$ type 2 sources, as pointed out by Szokoly et al. (2004). The number of type 2 candidates we find has therefore to be considered a lower limit.

One commonly adopted definition of absorbed source is $N_{\mathrm{H}}>10^{22} \mathrm{~cm}^{-2}$, where $N_{\mathrm{H}}$ is the intrinsic absorption at the redshift of the source. We discuss below how this compares with our definition.

\subsection{Finding the optical counterparts}

The optical counterparts to the X-ray sources were selected by cross-matching the absorbed X-ray sources with the GOODS ACS catalogues (29599 sources in the CDF-S, 32048 in the HDF-N). The GOODS catalogues contain sources that were detected in the $z$-band, with $B V i$ photometry in matched apertures (Giavalisco et al. 2004b). We checked for possible offsets between the optical and X-ray astrometry by crossmatching the full (CDF-S and HDF-N) Alexander catalogues with the GOODS catalogues using a threshold radius of $1.25^{\prime \prime}$ (see below). In the HDF-N we detected a systematic shift of $-0.029^{\prime \prime}$ in RA, and $-0.297^{\prime \prime}$ in declination of the Alexander positions with respect to the GOODS positions, and we applied this correction to the Alexander coordinates before doing the cross-match.

Note that the ACS image areas for the CDF-S and the HDF-N are both smaller than (and are completely within) their respective Chandra image fields. 546 out of the 829 sources in the Alexander catalogue (CDF-S: 222/326, HDF-N: 324/503) 
fall within the ACS image area. Of the 294 absorbed sources, 203 (CDF-S: 77, HDF-N: 126) fall within the ACS image area.

To find the optical counterparts of the absorbed X-ray sources, we initially search for optical sources that lie within a relatively large threshold radius of $3.5^{\prime \prime}$ (corresponding to the maximal $3 \sigma$ positional uncertainty of the X-ray positions) around each X-ray source. This was done using the cross match facility in the AVO prototype tool using the "best match" mode.

We find 195 "best" matches, 76 in the CDF-S and 119 in the HDF-N. So, in the CDF-S, all but one of the absorbed sources in the ACS image area have optical counterparts. The remaining source, Alexander ID 213, falls within the 10" disk of a bright galaxy. In the HDF-N there are 7 absorbed sources within the image area that do not have an optical counterpart (these 7 sources do however have optical matches in Barger et al. (2003), although we note that most of these are below the $5 \sigma$ limit of their catalogue).

Since the $3.5^{\prime \prime}$ radius is large relative to the median positional error, and given the optical source density the initial cross match inevitably includes a number of false and multiple matches. To limit our sample to good matches, we use the criterion that the cross match distance be less than the combined optical and X-ray $3 \sigma$ positional uncertainty for each individual match. Applying this distance/error $<1$ criterion we limit the number of matches to 168 (CDF-S: 65, HDF-N: 103). These matches are all within a much smaller radius than our initial 3.5" threshold, with most of the distance/error $<1$ matches being within 1.25" (and two matches at 1.4 and 1.5").

Considering not only the best match but also the multiple matches within a threshold radius of 1.25" (and distance/error < 1) we find 189 matches (CDF-S: 67, HDF-N: 122 ) to the $168 \mathrm{X}$-ray sources. This means that our method of only considering the best matches does discard 21 possibly valid matches in preference for a closer match.

The number of false matches we expect to have, as detailed in Appendix A, is small, between 8 and $15 \%$.

Our method requires $R$ magnitudes in order to estimate the $\mathrm{X}$-ray luminosity via the Fiore et al. correlation. The ACS band closest to the $R$ band is the $i$ band. We then convert the ACS $i$ magnitudes to the $R$ band assuming $\left(R-i_{\text {ACS }}\right)=0.5$, which is the typical value we derive for previously known type 2 AGN in the GOODS fields (see Sect. 6.1). The $R$ band flux, $f(R)$, was then computed by converting $R$ magnitudes to specific fluxes and then multiplying by the width of the $R$ filter (Zombeck 1990), as in Fiore et al. (2003).

\subsection{Estimating the $X$-ray power}

As discussed above, previously classified sources and their spectroscopic redshifts are available from two catalogues: Szokoly et al. (2004) for the CDF-S and Barger et al. (2003) for the HDF-N. For these sources we derived the $2-8 \mathrm{keV}$ $\mathrm{X}$-ray power, $L_{2-8}$, using our adopted cosmology and assuming $\alpha_{\mathrm{x}}=0.8$ for the k-correction (Fiore et al. 2003). No absorption correction was applied, which means that the intrinsic powers of our sources could be larger. As we are dealing with the hard
X-ray band, however, this is probably not going to make much difference, as pointed out by Fiore et al. (2003).

The known sources in the HDF-N were easily identified in our list of candidates because Barger et al. (2003) used the same Alexander et al. (2003) catalogue of X-ray sources. All 103 HDF-N sources that passed the distance/error $<1$ criterion are listed in Barger et al. (2003), 56 of which have spectroscopic redshifts, leaving 47 unclassified HDF-N sources. For the CDF-S Szokoly et al. (2004) utilised the X-ray catalogue of Giacconi et al. (2002) (which is derived from the same data as Alexander et al. 2003, but with somewhat different source extraction procedures), and list only the positions of their optical counterparts as determined from VLT FORS1 $R$-band imaging. To establish which CDF-S candidates are already listed in the Szokoly et al. (2004) catalogue we cross matched the GOODS ACS optical positions of our candidates with the Szokoly optical positions, and inspected all cases where there were multiple or possibly confused matches. Out of the 65 CDF-S sources which passed the distance/error $<1$ criterion, 54 are listed in Szokoly et al. (2004), and 44 of these have spectroscopic redshifts. This leaves a total of 68 (CDF-S: 21, HDF-N: 47) unclassified candidates.

For the unclassified sources we estimated the X-ray power as follows: we first derived the $f(2-10 \mathrm{keV}) / f(R)$ flux ratio, and then estimated the X-ray power from the correlation found by Fiore et al. (2003), namely $\log L_{2-10}=$ $\log f(2-10 \mathrm{keV}) / f(R)+43.05$ (Fiore, p.c., see their Fig. 5). Note that this correlation has an rms of $\sim 0.5$ dex in X-ray power and that, since the X-ray powers in the Fiore et al. (2003) correlation have been corrected for absorption, the estimated powers are already automatically corrected.

We stress that our estimated X-ray powers reach $\sim 10^{45} \mathrm{erg} / \mathrm{s}$ and therefore fall within the range of the Fiore et al. (2003) correlation.

\subsection{Finding the type 2 AGN candidates}

The work of Szokoly et al. (2004) has shown that absorbed, type $2 \mathrm{AGN}$ are characterized by $H R \geq-0.2$. It is also well known that normal galaxies, irrespective of their morphology, have X-ray powers that reach, at most, $L_{\mathrm{x}} \lesssim 10^{42} \mathrm{erg} / \mathrm{s}$ (e.g., Forman et al. 1994; Shapley et al. 2001; Cohen 2003). Therefore, any X-ray source with $H R \geq-0.2$ and $L_{\mathrm{x}}>$ $10^{42} \mathrm{erg} / \mathrm{s}$ should be an obscured AGN. Furthermore, following Szokoly et al. (2004), any such source having $L_{\mathrm{x}}>10^{44} \mathrm{erg} / \mathrm{s}$ will qualify as a type 2 QSO.

It is a well-established fact that, at a given optical magnitude, galaxies are also weaker X-ray emitters than AGN, that is they have much smaller X-ray-to-optical flux ratios, typically $\lesssim 0.1$ (Maccacaro et al. 1988). Indeed, the Fiore et al. (2003) correlation implies that $L_{\mathrm{x}}<10^{42} \mathrm{erg} / \mathrm{s}$ corresponds to $f(2-10 \mathrm{keV}) / f(R)<0.1$, so a selection based on X-ray-tooptical flux ratio would produce the same results.

\section{Results}

Our selection criteria have produced 68 type 2 AGN candidates, based on the definitions and the method described above. 
Table 1. Type 2 AGN candidates, HDF-N.

\begin{tabular}{|c|c|c|c|c|c|c|c|c|}
\hline GOODS ID & A03 & RA & DEC & $\begin{array}{l}\text { OFFSET } \\
\operatorname{arcsec}\end{array}$ & $\begin{array}{c}f(0.5-8 k e V) \\
10^{-15} \mathrm{erg} \mathrm{cm}^{-2} \mathrm{~s}^{-1}\end{array}$ & HR & $i$ & $\begin{array}{c}\log L_{\mathrm{x}}(2-8 k e V) \\
\mathrm{erg} / \mathrm{s}\end{array}$ \\
\hline J123542.91+621144.6 & 24 & 123542.98 & +621144.4 & 0.50 & 1.51 & 0.23 & $27.51 \pm 0.20$ & 44.8 \\
\hline $\mathrm{J} 123556.10+621219.6$ & 48 & 123556.14 & +621219.2 & 0.33 & 18.7 & 0.78 & $22.50 \pm 0.02$ & 43.9 \\
\hline $\mathrm{J} 123606.32+621233.2$ & 75 & 123606.38 & +621232.2 & 0.84 & 0.96 & 0.14 & $25.58 \pm 0.07$ & 43.8 \\
\hline $\mathrm{J} 123606.86+621021.7$ & 79 & 123606.84 & +621021.4 & 0.11 & 0.74 & 1.00 & $24.56 \pm 0.07$ & 43.4 \\
\hline $\mathrm{J} 123611.78+621015.0$ & 92 & 123611.80 & +621014.5 & 0.28 & 2.50 & 0.69 & $25.94 \pm 0.18$ & 44.5 \\
\hline $\mathrm{J} 123613.73+620800.5$ & 99 & 123613.73 & +620800.2 & 0.03 & 1.62 & 0.09 & $25.54 \pm 0.17$ & 44.0 \\
\hline $\mathrm{J} 123614.46+621045.5$ & 102 & 123614.45 & +621045.0 & 0.15 & 3.63 & 0.54 & $24.70 \pm 0.06$ & 44.1 \\
\hline $\mathrm{J} 123616.05+621108.1$ & 108 & 123616.03 & +621107.7 & 0.18 & 16.07 & 0.27 & $25.16 \pm 0.10$ & 44.9 \\
\hline $\mathrm{J} 123616.12+621514.0$ & 109 & 123616.11 & +621513.7 & 0.01 & 1.02 & -0.09 & $26.22 \pm 0.15$ & 44.1 \\
\hline $\mathrm{J} 123620.88+621415.8$ & 123 & 123620.91 & +621415.5 & 0.20 & 0.96 & 0.46 & $24.76 \pm 0.08$ & 43.5 \\
\hline $\mathrm{J} 123621.96+621603.6$ & 129 & 123621.94 & +621603.8 & 0.48 & 1.22 & 1.00 & $27.33 \pm 0.27$ & 44.7 \\
\hline J123622.59+621306.5 & 133 & 123622.57 & +621306.2 & 0.10 & 0.28 & -0.13 & $24.70 \pm 0.04$ & 42.9 \\
\hline J123622.64+621630.2 & 135 & 123622.66 & +621629.8 & 0.23 & 1.02 & 1.00 & $24.66 \pm 0.07$ & 43.6 \\
\hline $\mathrm{J} 123624.81+621744.2$ & 143 & 123624.78 & +621743.7 & 0.32 & 0.47 & 1.00 & $25.78 \pm 0.14$ & 43.7 \\
\hline $\mathrm{J} 123627.60+621158.8$ & 152 & 123627.75 & +621158.4 & 1.10 & 2.22 & 0.50 & $23.87 \pm 0.03$ & 43.6 \\
\hline J123634.46+620942.3 & 181 & 123634.48 & +620941.8 & 0.32 & 0.57 & -0.10 & $27.12 \pm 0.20$ & 44.1 \\
\hline J123634.62+621422.1 & 185 & 123634.64 & +621422.1 & 0.32 & 0.12 & 0.33 & $26.23 \pm 0.14$ & 43.3 \\
\hline $\mathrm{J} 123635.28+621152.2$ & 188 & 123635.26 & +621151.6 & 0.32 & 0.40 & -0.07 & $24.66 \pm 0.05$ & 43.0 \\
\hline $\mathrm{J} 123637.12+620636.7$ & 198 & 123637.26 & +620637.5 & 1.48 & 0.25 & 1.00 & $27.01 \pm 0.17$ & 43.9 \\
\hline $\mathrm{J} 123644.13+621245.2$ & 228 & 123644.12 & +621244.5 & 0.39 & 0.08 & 1.00 & $24.99 \pm 0.10$ & 42.9 \\
\hline J123646.58+620857.0 & 242 & 123646.50 & +620856.9 & 0.59 & 0.51 & 1.00 & $23.75 \pm 0.04$ & 43.0 \\
\hline $\mathrm{J} 123647.94+621020.6$ & 246 & 123647.94 & +621019.9 & 0.41 & 1.93 & 0.54 & $26.83 \pm 0.27$ & 44.7 \\
\hline $\mathrm{J} 123651.28+621052.0$ & 266 & 123651.28 & +621051.5 & 0.20 & 2.42 & 0.77 & $24.06 \pm 0.04$ & 43.7 \\
\hline $\mathrm{J} 123653.17+621947.8$ & 276 & 123653.07 & +621947.8 & 0.69 & 0.64 & 1.00 & $27.00 \pm 0.18$ & 44.4 \\
\hline $\mathrm{J} 123654.11+620835.4$ & 281 & 123654.13 & +620835.1 & 0.17 & 0.66 & 1.00 & $22.50 \pm 0.01$ & 42.6 \\
\hline J123654.59+621111.8 & 284 & 123654.58 & +621110.6 & 0.88 & 0.94 & 0.20 & $26.55 \pm 0.10$ & 44.2 \\
\hline $\mathrm{J} 123658.77+621022.7$ & 303 & 123658.79 & +621022.2 & 0.30 & 1.56 & 0.69 & $24.88 \pm 0.07$ & 43.8 \\
\hline $\mathrm{J} 123700.45+621509.2$ & 312 & 123700.45 & +621508.8 & 0.08 & 1.80 & -0.19 & $25.17 \pm 0.08$ & 43.9 \\
\hline $\mathrm{J} 123702.44+621926.0$ & 321 & 123702.43 & +621926.1 & 0.44 & 3.97 & -0.18 & $26.93 \pm 0.24$ & 44.9 \\
\hline $\mathrm{J} 123706.88+622225.6$ & 341 & 123706.72 & +622224.5 & 1.35 & 0.39 & 1.00 & $25.22 \pm 0.09$ & 43.3 \\
\hline $\mathrm{J} 123707.21+621408.5$ & 347 & 123707.20 & +621407.9 & 0.30 & 0.98 & 0.23 & $25.39 \pm 0.11$ & 43.8 \\
\hline $\mathrm{J} 123709.93+622259.4$ & 362 & 123709.88 & +622258.7 & 0.50 & 5.21 & 0.64 & $23.90 \pm 0.07$ & 43.9 \\
\hline $\mathrm{J} 123711.34+621330.9$ & 366 & 123711.38 & +621330.8 & 0.38 & 0.56 & 0.11 & $24.38 \pm 0.07$ & 43.1 \\
\hline $\mathrm{J} 123712.00+621325.9$ & 368 & 123712.04 & +621325.7 & 0.35 & 0.92 & 1.00 & $25.11 \pm 0.10$ & 43.7 \\
\hline $\mathrm{J} 123712.04+621212.3$ & 369 & 123712.09 & +621211.3 & 0.84 & 0.38 & 0.33 & $25.94 \pm 0.14$ & 43.6 \\
\hline J123713.84+621458.7 & 375 & 123713.90 & +621458.0 & 0.60 & 1.11 & 1.00 & $26.50 \pm 0.19$ & 44.4 \\
\hline $\mathrm{J} 123714.68+621839.7$ & 380 & 123714.60 & +621839.5 & 0.57 & 0.48 & 1.00 & $25.62 \pm 0.06$ & 43.6 \\
\hline $\mathrm{J} 123720.34+621353.6$ & 402 & 123720.34 & +621353.1 & 0.20 & 1.35 & 1.00 & $23.02 \pm 0.02$ & 43.0 \\
\hline J123723.99+621304.7 & 412 & 123724.00 & +621304.3 & 0.13 & 6.07 & 0.49 & $24.62 \pm 0.04$ & 44.3 \\
\hline $\mathrm{J} 123726.51+622026.8$ & 423 & 123726.51 & +622026.8 & 0.27 & 1.40 & 0.17 & $24.99 \pm 0.07$ & 43.8 \\
\hline $\mathrm{J} 123726.85+621747.9$ & 424 & 123726.77 & +621747.8 & 0.56 & 1.68 & 0.17 & $26.10 \pm 0.18$ & 44.3 \\
\hline $\mathrm{J} 123731.13+621030.1$ & 430 & 123731.13 & +621030.0 & 0.22 & 0.54 & -0.06 & $25.31 \pm 0.10$ & 43.4 \\
\hline J123731.54+621305.9 & 431 & 123731.55 & +621306.1 & 0.54 & 0.40 & -0.18 & $26.04 \pm 0.13$ & 43.6 \\
\hline $\mathrm{J} 123734.09+621624.8$ & 434 & 123733.98 & +621624.0 & 0.93 & 0.82 & 1.00 & $25.42 \pm 0.08$ & 43.8 \\
\hline $\mathrm{J} 123737.06+621834.5$ & 445 & 123737.04 & +621834.4 & 0.24 & 4.43 & -0.13 & $27.18 \pm 0.29$ & 45.1 \\
\hline $\mathrm{J} 123737.95+621713.9$ & 447 & 123737.90 & +621713.3 & 0.42 & 0.86 & 0.22 & $24.78 \pm 0.10$ & 43.5 \\
\hline $\mathrm{J} 123804.23+621526.0$ & 486 & 123804.11 & +621525.2 & 1.00 & 2.77 & 0.43 & $22.58 \pm 0.03$ & 43.2 \\
\hline
\end{tabular}

These sources are listed in Tables 1 (HDF-N) and 2 (CDF-S). Table 1 gives the GOODS ID in Col. 1, the Alexander et al. (2003) IDs in Col. 2, the X-ray position in Cols. 3-4, the offset between the X-ray and optical positions in arcseconds in Col. 5, the full-band $(0.5-8 \mathrm{keV}) \mathrm{X}$-ray flux in Col. 6, the hardness ratio in Col. 7, the ACS $i$ magnitude in Col. 8, the estimated $\mathrm{X}$-ray power in Col. 9. Table 2 gives, in addition, the Szokoly et al. (2004) IDs in Col. 2, and the remaining columns are then shifted by one.

Out of our candidates, 31 satisfy the further requirement $L_{2-10}>10^{44} \mathrm{erg} / \mathrm{s}$ and therefore qualify as QSO 2 candidates. We note that the distribution of estimated X-ray power covers the range $5 \times 10^{42}-2 \times 10^{45} \mathrm{erg} / \mathrm{s}$ and peaks around $10^{44} \mathrm{erg} / \mathrm{s}$ (see Fig. 5, dashed line). The number of QSO 2 candidates, therefore, is very sensitive to the dividing line between low- and high-luminosity AGN, which is clearly arbitrary and cosmology dependent. For example, if one defines as QSO 2 all sources with $L_{2-10}>5 \times 10^{43} \mathrm{erg} / \mathrm{s}$, a value only a factor of 2 below the commonly used one and corresponding to the break in the AGN X-ray luminosity function (Norman et al. 2002), the number of such sources increases by $\sim 50 \%$. We also note that, based on the rms around the Fiore et al. (2003) correlation, the number of QSO 2 candidates fluctuates in the 13-54 region. The number of type $2 \mathrm{AGN}$, on the other hand, can only increase, as all our candidates have estimated $\log L_{2-10}>42.5$.

It is interesting that all candidates with $H R \geq-0.2$ have estimated X-ray power $L_{\mathrm{x}, \mathrm{est}}>10^{42} \mathrm{erg} / \mathrm{s}$, and therefore all qualify as AGN. Some previously known sources, however, do have X-ray powers below this value (see Sect. 6.1).

As expected, being still unidentified, our sources are very faint: their median ACS $i$ magnitude is $\sim 25.5$, which 
Table 2. Type 2 AGN candidates, CDF-S.

\begin{tabular}{|c|c|c|c|c|c|c|c|c|c|}
\hline GOODS ID & S04 & A03 & RA & DEC & $\begin{array}{l}\text { OFFSET } \\
\text { arcsec }\end{array}$ & $\begin{array}{c}f(0.5-8 \mathrm{keV}) \\
10^{-15} \mathrm{erg} \mathrm{cm}^{-2} \mathrm{~s}^{-1}\end{array}$ & HR & $\mathrm{i}$ & $\begin{array}{c}\log L_{\mathrm{x}}(2-8 k e V) \\
\mathrm{erg} / \mathrm{s}\end{array}$ \\
\hline J033205.35-274644.1 & & 50 & 033205.35 & -274644.1 & 0.04 & 3.37 & 0.58 & $26.11 \pm 0.19$ & 44.7 \\
\hline J033206.12-274927.8 & 259 & 52 & 033206.13 & -274927.7 & 0.16 & 4.43 & 0.64 & $23.81 \pm 0.05$ & 43.8 \\
\hline J033214.86-275103.8 & $82 \mathrm{a}$ & 100 & 033214.87 & -275103.8 & 0.17 & 1.74 & 0.02 & $25.82 \pm 0.16$ & 44.2 \\
\hline J033217.11-274136.9 & & 116 & 033217.11 & -274136.6 & 0.28 & 1.60 & 0.18 & $26.32 \pm 0.15$ & 44.4 \\
\hline J033219.95-274243.1 & 524 & 130 & 033219.96 & -274243.2 & 0.10 & 1.43 & 0.03 & $27.18 \pm 0.20$ & 44.7 \\
\hline J033221.78-274656.5 & & 138 & 033221.75 & -274657.0 & 0.66 & 0.15 & 1.00 & $26.63 \pm 0.24$ & 43.7 \\
\hline J033222.54-274603.8 & 145 & 145 & 033222.54 & -274603.9 & 0.06 & 4.74 & 0.32 & $24.59 \pm 0.04$ & 44.2 \\
\hline J033224.54-274010.4 & & 156 & 033224.55 & -274010.7 & 0.28 & 0.89 & 1.00 & $22.54 \pm 0.01$ & 42.8 \\
\hline J033224.85-275600.0 & & 159 & 033224.84 & -275600.0 & 0.08 & 5.61 & -0.05 & $23.74 \pm 0.04$ & 43.9 \\
\hline J033226.78-274604.0 & 518 & 175 & 033226.75 & -274604.3 & 0.49 & 0.74 & 0.11 & $27.44 \pm 0.35$ & 44.5 \\
\hline J033229.78-275328.4 & & 187 & 033229.84 & -275328.6 & 0.78 & 0.77 & 1.00 & $27.03 \pm 0.23$ & 44.4 \\
\hline J033232.16-274651.5 & 515 & 201 & 033232.17 & -274651.4 & 0.10 & 1.57 & 0.39 & $27.01 \pm 0.24$ & 44.7 \\
\hline J033233.28-274236.0 & & 206 & 033233.27 & -274236.1 & 0.21 & 4.01 & 0.43 & $25.51 \pm 0.11$ & 44.5 \\
\hline J033235.21-275318.0 & 148 & 218 & 033235.23 & -275317.8 & 0.37 & 3.19 & 0.08 & $27.10 \pm 0.27$ & 45.0 \\
\hline J033236.16-275037.1 & 609 & 223 & 033236.17 & -275037.2 & 0.22 & 0.68 & 1.00 & $25.40 \pm 0.11$ & 43.8 \\
\hline J033238.76-275121.6 & & 237 & 033238.78 & -275121.6 & 0.25 & 0.92 & 0.12 & $26.15 \pm 0.08$ & 44.1 \\
\hline J033239.05-274439.3 & & 241 & 033239.06 & -274439.1 & 0.25 & 2.64 & -0.11 & $26.64 \pm 0.19$ & 44.7 \\
\hline J033239.17-274832.4 & & 243 & 033239.17 & -274832.7 & 0.33 & 0.20 & 1.00 & $26.27 \pm 0.13$ & 43.8 \\
\hline J033243.04-274845.1 & 256 & 255 & 033243.05 & -274845.0 & 0.19 & 4.05 & 0.69 & $25.15 \pm 0.06$ & 44.3 \\
\hline J033246.34-274632.0 & 147 & 268 & 033246.35 & -274631.9 & 0.13 & 7.03 & 0.64 & $24.95 \pm 0.11$ & 44.5 \\
\hline J033247.06-275333.5 & & 272 & 033247.05 & -275333.3 & 0.26 & 3.02 & -0.03 & $26.21 \pm 0.13$ & 44.6 \\
\hline
\end{tabular}

corresponds to $R \sim 26$ (compare this to the $R \sim 22$ typical of the CDF-S sources with redshift determination). The QSO 2 candidates are even fainter, with median $i$ magnitude $\sim 26.3(R \sim 26.8)$.

It is important to notice that 6 of the 21 sources in Table 2 have actually been observed by Szokoly et al. (2004, see their Fig. 5) but either their spectrum is featureless (4 sources) or the continuum is so weak that no extraction could be made (2 sources).

\subsection{Testing our estimated $X$-ray powers}

To check the reliability of our estimated X-ray powers we compared the predicted and observed luminosities for the already known sources. The Fiore et al. (2003) correlation is valid for non-type $1 \mathrm{AGN}$ and galaxies so we did the comparison for these sources. Namely, we excluded the type 1 sources from the Szokoly et al. (2004) catalogue and the sources with broad lines (type "B") in Barger et al. (2003). Moreover, we only considered sources with quality flag $\geq 2.0$ in the former catalogue and excluded objects with less secure redshifts (type "s") in the latter, to have reliable redshifts and therefore powers. This leaves us with 122 objects. For these, we find that $L_{\mathrm{x}, \text { est }} \propto L_{\mathrm{x}}^{0.93 \pm 0.06}$, where the slope is the mean ordinary least-squares slope (Isobe et al. 1990). The mean values of the estimated $\left(\left\langle\log L_{\mathrm{x}, \text { est }}\right\rangle=\right.$ $42.57 \pm 0.08)$ and observed $\left(\left\langle\log L_{\mathrm{x}}\right\rangle=42.49 \pm 0.09\right)$ luminosities, are consistent. This shows that our method works for non-type 1 AGN and can then be safely applied to optically unidentified but absorbed sources.

It is tempting to use our estimated X-ray powers together with the observed fluxes to derive redshifts for our type 2 candidates. We can first check how reliable these are by comparing them with the spectroscopic redshifts available for the known non-type 1 AGN described above. We follow standard procedures (see, e.g., Mobasher et al. 2004) and quantify the reliability of the estimated redshifts, $z_{\text {est }}$, by measuring the fractional error for each galaxy, $\Delta \equiv\left(z_{\text {est }}-z_{\text {spec }}\right)\left(1+z_{\text {spec }}\right)$.
We find a median error, $\langle\Delta\rangle=0.005$, an rms scatter, $\sigma(\Delta) \sim$ 0.29 , and a rate of "catastrophic" outliers, $\eta$, defined as the fraction of the full sample that has $|\Delta|>0.2, \sim 19 \%$. Clipping these outliers gives $\langle\Delta\rangle=-0.03$ and $\sigma(\Delta) \sim 0.1$. For comparison, Mobasher et al. (2004) have used extensive multiwavelength photometric data to estimate photometric redshifts for a sample of 434 galaxies with spectroscopic redshifts in the CDF-S. They find $\langle\Delta\rangle=-0.01, \sigma(\Delta) \sim 0.1$, and $\eta \sim 2.4-10 \%$, depending on the subsample considered. Excluding the outliers they find values of $\sigma(\Delta) \sim 0.05$. Given the simplicity of our method, these results are certainly encouraging and show that our estimates should at least provide a rough idea of the redshifts at which we might expect our sources to be.

Applying this method to our type 2 AGN candidate list we find a mean of $\left\langle z_{\text {est }}\right\rangle \sim 2.9$ (median 2.6). The QSO 2 have, as expected, significantly higher estimated redshifts $\left\langle z_{\text {est }}\right\rangle \sim 3.7$ compared to $\left\langle z_{\text {est }}\right\rangle \sim 2.2$ for the non-QSO candidates.

We can also use the ACS four band photometry to constrain the redshifts of our sources. Cristiani et al. (2004) have discussed a set of four optical criteria using the $i-z, B-V$, and $V-i$ colours (a variation of the "B-dropout" technique) to select AGN in the redshift range $3.5 \lesssim z \lesssim 5.2$. By applying the same criteria to our candidates we find 9 such sources. Their average estimated redshift is $\left\langle z_{\text {est }}\right\rangle \sim 3.6$, and all but one sources have $2.8<z_{\text {est }}<5.4$. Again, this shows that our estimated redshifts are, overall, relatively robust.

These sources are listed in Table 3, which gives the GOODS ID in Col. 1, the ACS $i$ magnitude in Col. 2, followed by the $i-z, V-i$ and $B-V$ colours (all in the AB system) in Cols. 3-5.

\subsection{Testing our method}

Out of the 9 QSO 2 previously known (see Sect. 6), our method has rediscovered them all as type $2 \mathrm{AGN}, 8 / 9$ with $L_{\mathrm{x}, \text { est }}>8 \times$ $10^{43} \mathrm{erg} / \mathrm{s}$ (the ninth one having $L_{\mathrm{x}, \text { est }} \sim 4 \times 10^{42} \mathrm{erg} / \mathrm{s}$ ). Out of 

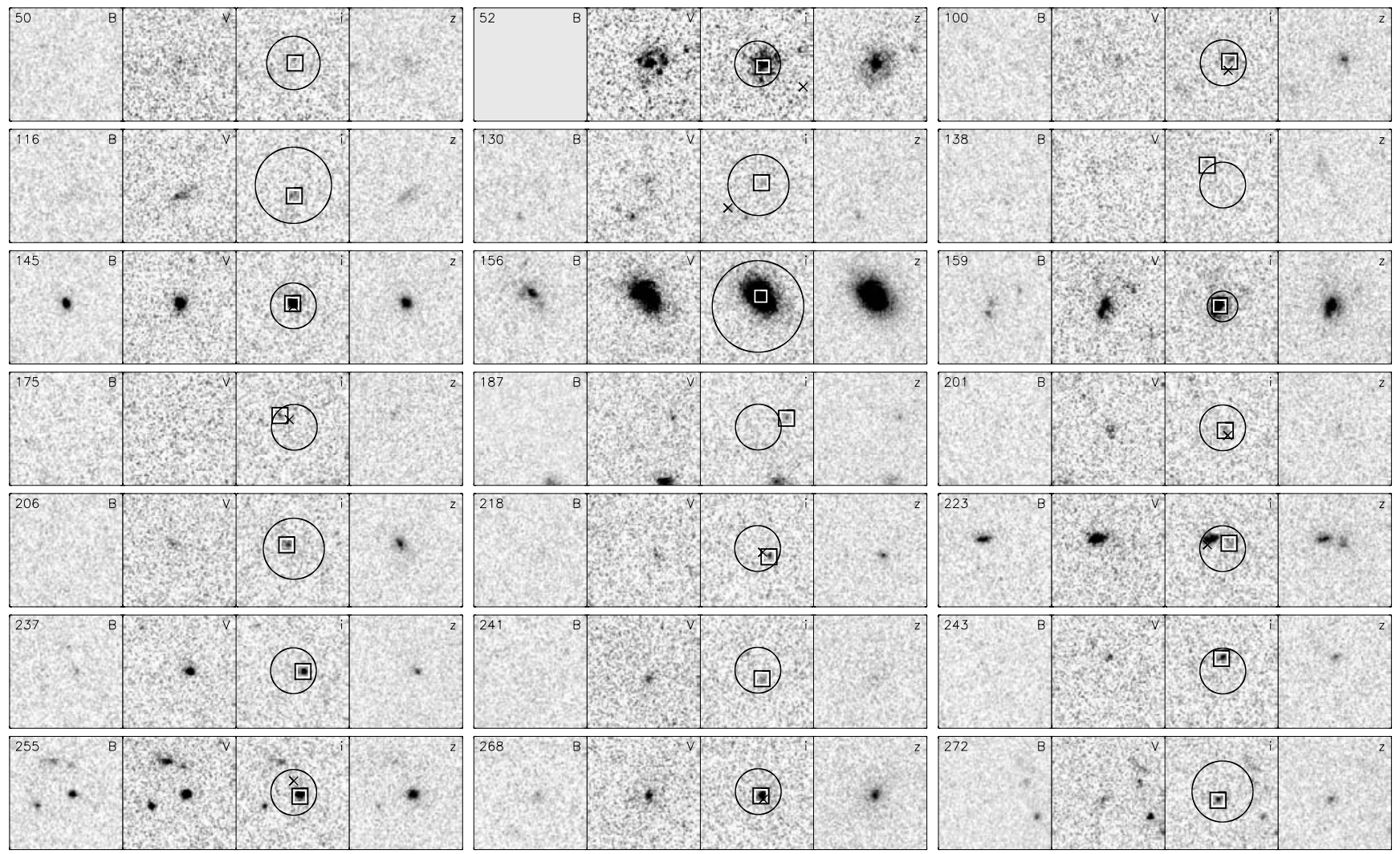

Fig. 2. CDF-S Cutouts. $B, V, i$, and $z$ ACS image cutouts are displayed for all the type 2 AGN candidate in the CDF-S. Each image is $3^{\prime \prime} \times 3^{\prime \prime}$ in size, with north up and east to the left. The Alexander identification number is shown in the top left of each panel, and the circles drawn on the $i$-band images indicate the Alexander X-ray positions, with the radius indicating the $90 \%$ positional error. The (black and white) square symbols indicate the positions of the GOODS source which have been matched to the X-ray positions. The X symbols indicate the positions of any corresponding sources in the Szokoly catalogue.

Table 3. B dropouts.

\begin{tabular}{lcccr}
\hline \hline GOODS ID & $i$ & $i-z$ & $V-i$ & $B-V$ \\
\hline J033226.78-274604.0 & 27.44 & 0.37 & 3.21 & -1.36 \\
J033232.16-274651.5 & 27.01 & 0.27 & 0.43 & 1.25 \\
J033238.76-275121.6 & 26.15 & 0.04 & 0.23 & 1.41 \\
J033239.05-274439.3 & 26.64 & 0.26 & 0.23 & 4.41 \\
J123611.78+621015.0 & 25.94 & 0.71 & 2.46 & $>3.0$ \\
J123627.59+621158.8 & 23.86 & 0.24 & 0.69 & 1.25 \\
J123634.46+620942.3 & 27.12 & 0.28 & 1.53 & $>3.0$ \\
J123714.68+621839.7 & 25.62 & 0.13 & 0.44 & 1.87 \\
J123731.54+621305.9 & 26.04 & -0.13 & 0.46 & 1.39 \\
\hline
\end{tabular}

the 29 type 2 AGN with $L_{2-8}>10^{42} \mathrm{erg} / \mathrm{s}$ in the Szokoly et al. (2004) catalogue, 28 have $L_{\mathrm{x}, \text { est }}>10^{42} \mathrm{erg} / \mathrm{s}$ and therefore fulfil our selection criteria, while the last one is barely below with $L_{\mathrm{x}, \text { est }} \sim 7 \times 10^{41} \mathrm{erg} / \mathrm{s}$.

During the completion of this work three redshift databases covering the CDF-S and HDF-N became public. Namely: the ESO/GOODS FORS2 spectroscopy data (Vanzella et al. $2004^{9}$ ), the VIMOS VLT Deep Survey (VVDS;

\footnotetext{
${ }^{9}$ http://archive.eso.org/wdb/wdb/vo/goods/form
}

Le Fèvre et al. 2004 ${ }^{10}$ ) and the Team Keck Treasury Redshift Survey (TKRS; Wirth et al. 2004 ${ }^{11}$ ). We cross-matched our candidate list with these surveys using the AVO prototype, finding only one match, namely GOODS J123556.10+621219.6, with a quite featureless spectrum and redshift $z=0.9585$ (our estimated redshift based on its X-ray power and flux is $z=$ $0.93)$. Given the limits of the three surveys, that is $z_{\mathrm{AB}}<24.5$, $I_{\mathrm{AB}} \leq 24$, and $R \leq 24.4$ respectively, this is not surprising. The only match we found, in fact, is the brightest of our northern candidates.

\subsection{Image cutouts}

Image cutouts of the ACS BViz data centred on each of the new AGN type 2 candidates were generated using the AVO prototype. This was done by sending requests to the Aladin image server which remotely extracts the required image sections in the four bands from a database containing the GOODS data. The hierarchical description of the ACS images in terms of the IDHA data model, and advanced protocols for sending detailed requests to the server makes this process more efficient and flexible than traditional image servers. Figures 2 and 3 display $3^{\prime \prime} \times 3^{\prime \prime}$ sections of the cutouts in all four bands for the

\footnotetext{
${ }^{10}$ http://cencosw.oamp.fr/

11 http://www2 . keck .hawaii.edu/science/tksurvey/
} 

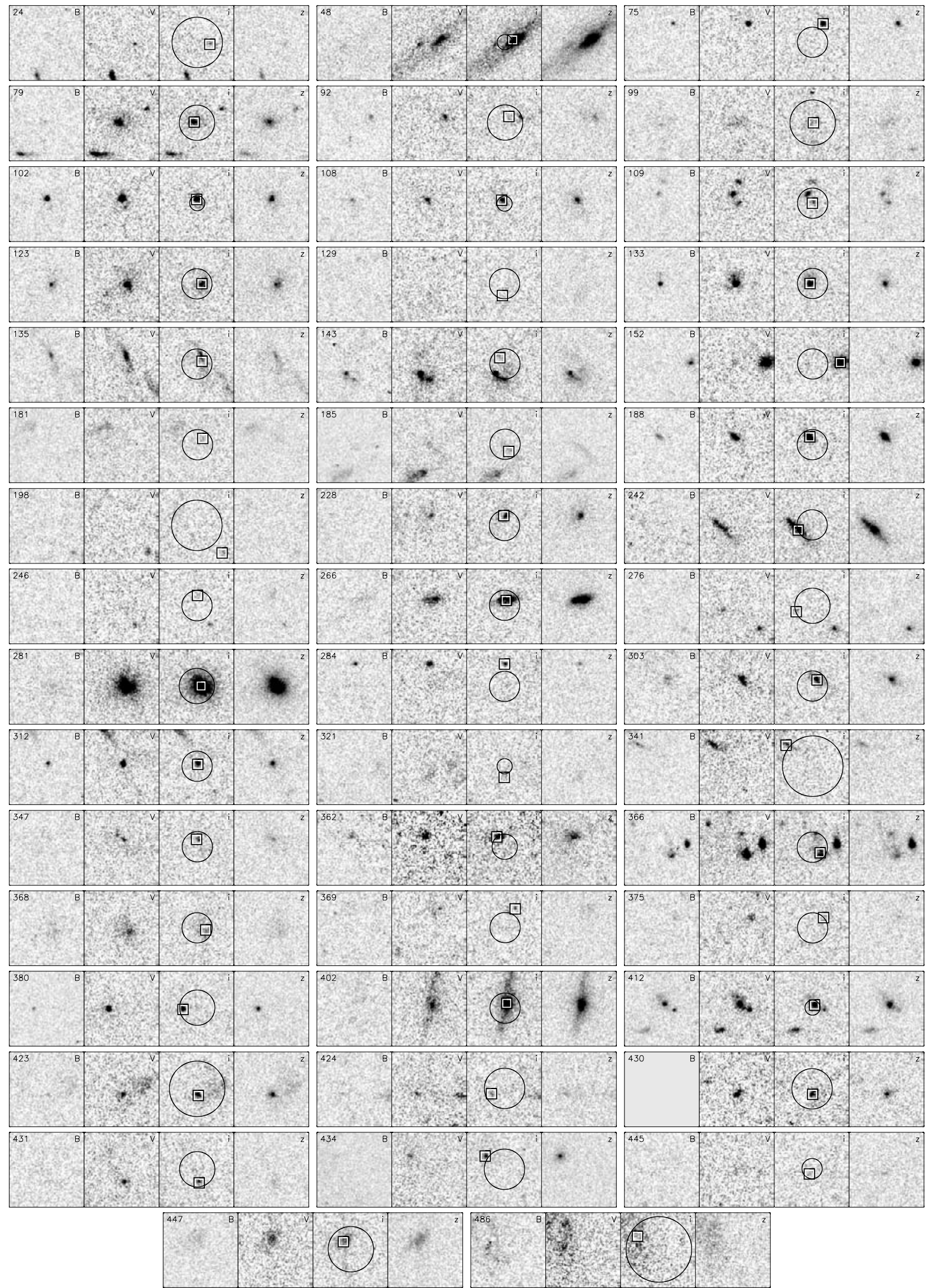

Fig. 3. CDFN Cutouts. $B, V, i$, and $z$ ACS image cutouts are displayed for all the type 2 AGN candidate in the CDFN. Each image is $3^{\prime \prime} \times 3^{\prime \prime}$ in size, with north up and east to the left. The Alexander identification number is shown in the top left of each panel, and the circles drawn on the $i$-band images indicates the Alexander X-ray positions, with the radius indicating the $90 \%$ positional error. The (black and white) square symbols indicate the positions of the GOODS sources which have been matched to the X-ray positions. 
Table 4. Type 2 AGN candidates, UDF.

\begin{tabular}{cccccccc}
\hline \hline S04 & A03 & UDF ID & RA & Dec & $\begin{array}{c}\text { OFFSET } \\
\operatorname{arcsec}\end{array}$ & & cog \\
& & & & & & erg/s \\
\hline 515 & 201 & 7326 & 033232.17 & -274651.49 & 0.10 & $26.869 \pm 0.021$ & 44.6 \\
& 243 & 1441 & 033239.19 & -274832.87 & 0.31 & $28.328 \pm 0.046$ & 44.7 \\
256 & 255 & 1025 & 033243.04 & -274845.08 & 0.18 & $25.153 \pm 0.006$ & 44.3 \\
\hline
\end{tabular}

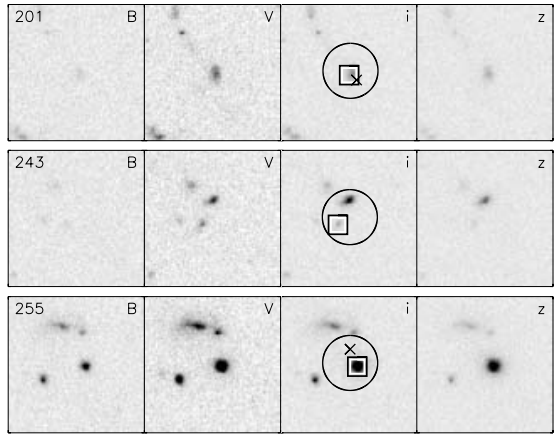

Fig. 4. CDF-S UDF Cutouts. $B, V, i$, and $z$ ACS image cutouts are displayed for the type 2 AGN candidates that fall within the area of the UDF. The (black and white) square symbols indicate the positions of the UDF sources in the UDF catalogue.The $\times$ symbols indicate the positions of any corresponding sources in the Szokoly catalogue.

CDF-S and HDF-N respectively. Each image is shown with a linear grey-scale over the range of minimum to maximum image values of the individual $3^{\prime \prime} \times 3^{\prime \prime}$ image sections. The $90 \%$ error circle of the Alexander et al. (2003) catalogue X-ray positions is overlaid on the $i$ band images (since our method used the $i$ band), along with the GOODS catalogue position (black/white squares). The Szokoly et al. (2004) optical positions (X symbols) are also shown for the CDF-S sources (Fig. 2). Note that in a few cases the position of the GOODS optical counterpart is outside the X-ray $90 \%$ error circle. These are still valid matches in terms of the distance/error parameter (see Sect. 4.4), which limits matches to within the $3 \sigma$ total positional uncertainty which is $\sim 1.8$ times larger than the $90 \%$ radius.

Our sources cover a large spectrum of morphologies, ranging from extended, low-surface brightness to point-like. It is interesting to note, however, that all QSO 2 candidates are point-like.

\subsection{Hubble Ultra Deep Field data}

A number of sources also fall within the Hubble Ultra Deep Field (UDF; Beckwith et al. 2004). The UDF provides extremely deep ACS Wide Field Channel BViz imaging (proposal IDs 9978 and 10086$)$ of a single $5.25^{\prime} \times 5.25^{\prime}$ field centred on $03^{\mathrm{h}} 32^{\mathrm{m}} 39.0^{\mathrm{s}},-27^{\circ} 47^{\prime} 29.1^{\prime \prime}(\mathrm{J} 2000)$ which lies within the GOODS CDF-S field. Five of the absorbed sources fall within this field, and 3 of these (A03 201, 243 and 255) are in our list of unclassified type 2 AGN candidates (Table 2). For these sources we cross matched the X-ray positions with the
UDF version $1.0 \mathrm{BViz} i$-band detected catalogues ${ }^{12}$ to find the UDF optical counterparts. We then used the UDF $i$ band magnitudes to estimate the X-ray power using the Fiore et al. (2003) correlation as described above. The estimated X-ray powers, and UDF $i$ band magnitudes for these 3 sources are listed in Table 4 and UDF image cutouts overlaid with the UDF, Alexander et al. (2003), and Szokoly et al. (2004) positions are shown in Fig. 4. Note that for one source, A03 243, we find a different, fainter, optical counterpart than was found using the GOODS catalogue, resulting in a larger estimated X-ray power. Classically, one would have solved this dilemma by obtaining a spectrum of both sources. However, given the faintness of the two candidates $(i \sim 26.3$ and 28.3), this is unlikely to happen in the near future. (The two previously classified sources that fall within the UDF are A03 245 (QSO-2) and 242 (AGN-1).)

Note that none of our candidates have spectra obtained with the ACS High Resolution Channel grism ${ }^{13}$ as part of the UDF observing campaign.

\section{Discussion}

\subsection{Previously known type 2 AGN}

We defined a sample of previously known type 2 AGN in the GOODS ACS fields as follows. In the HDF-N, we selected absorbed $(H R \geq-0.2)$ sources without broad lines (type " $\mathrm{B}$ " in Barger et al. 2003) having X-ray power $L_{2-8}>10^{42} \mathrm{erg} / \mathrm{s}$ (see Sect. 4.4). In the CDF-S we initially selected sources with "AGN-2" or "QSO-2" classifications in Szokoly et al. (2004). We then applied the further criteria that $L_{2-8}>10^{42} \mathrm{erg} / \mathrm{s}$ and $H R \geq-0.2$ in order to be consistent with the HDF-N sample. Successive power and $H R$ cuts exclude 2 and 3 sources respectively, with a further 3 sources excluded because they have no counterpart in the Alexander et al. (2003) catalogue (which is necessary for consistent definitions of $H R$ and $L_{2-8}$ ). (Note that differences between our $H R$ values and those listed by Szokoly et al. 2004, can be explained by the different input catalogues produced by Giacconi et al. 2002 and Alexander et al. 2003.) These definitions lead to a total of 79 known type 2 AGN (CDF-S: 35, HDF-N: 44). These are listed in Tables 5 and 6, which give the Alexander et al. (2003) and Szokoly et al. (2004) (for the CDF-S) IDs, redshift, hard $\mathrm{X}$-ray power, and notes. Only 9 of these sources can be classified as QSO $2\left(L_{2-8}>10^{44} \mathrm{erg} / \mathrm{s}\right), 3$ of which have poor redshift determinations (two sources with type "s" flags in Barger et al. 2003, and one source with quality flag $=1$ in

\footnotetext{
12 http://www.stsci.edu/hst/udf

13 http://www.stecf.org/UDF/goodsdata.html
} 
Table 5. Previously Known Type 2 AGN, HDF-N.

\begin{tabular}{|c|c|c|c|}
\hline A03 & redshift & $\begin{array}{c}\log L_{\mathrm{x}}(2-8 \mathrm{keV}) \\
\mathrm{erg} / \mathrm{s}\end{array}$ & Notes $^{a}$ \\
\hline 72 & 0.936 & 42.73 & $\mathrm{~m}$ \\
\hline 76 & 0.637 & 42.71 & $\mathrm{~m}$ \\
\hline 82 & 0.681 & 42.52 & \\
\hline 83 & 0.459 & 42.41 & \\
\hline 90 & 1.140 & 43.33 & \\
\hline 106 & 1.017 & 42.31 & \\
\hline 121 & 0.520 & 42.27 & \\
\hline 122 & 1.338 & 42.90 & $\mathrm{~s}$ \\
\hline 127 & 1.014 & 43.27 & $\mathrm{~m}$ \\
\hline 142 & 0.747 & 42.47 & \\
\hline 150 & 0.762 & 42.82 & \\
\hline 157 & 1.264 & 43.89 & \\
\hline 158 & 1.013 & 43.02 & \\
\hline 160 & 0.847 & 42.90 & $\mathrm{~m}$ \\
\hline 163 & 0.485 & 42.72 & \\
\hline 164 & 0.953 & 42.92 & \\
\hline 170 & 0.680 & 42.44 & \\
\hline 171 & 1.995 & 43.63 & $\mathrm{~m}$ \\
\hline 187 & 0.847 & 42.27 & \\
\hline 190 & 2.005 & 43.76 & \\
\hline 191 & 0.560 & 42.79 & $\mathrm{~m}$ \\
\hline 201 & 1.020 & 42.74 & $\mathrm{~m}$ \\
\hline 217 & 0.518 & 42.15 & \\
\hline 229 & 1.487 & 42.23 & $\mathrm{~s}$ \\
\hline 240 & 0.961 & 43.95 & \\
\hline 259 & 1.609 & 44.48 & $\mathrm{~m}$ \\
\hline 261 & 0.902 & 42.15 & \\
\hline 267 & 0.401 & 42.15 & \\
\hline 278 & 1.023 & 42.92 & \\
\hline 330 & 3.406 & 43.43 & \\
\hline 352 & 0.936 & 42.47 & \\
\hline 373 & 0.475 & 42.25 & \\
\hline 384 & 1.019 & 43.45 & \\
\hline 390 & 1.146 & 44.04 & $\mathrm{~s}$ \\
\hline 398 & 2.638 & 44.05 & $\mathrm{~s}$ \\
\hline 405 & 0.978 & 42.96 & \\
\hline 409 & 2.240 & 43.61 & \\
\hline 413 & 0.474 & 42.60 & \\
\hline 439 & 0.636 & 42.36 & \\
\hline 441 & 0.634 & 42.24 & \\
\hline 442 & 0.852 & 42.33 & \\
\hline 448 & 1.238 & 43.32 & \\
\hline 462 & 0.511 & 42.03 & \\
\hline 468 & 0.911 & 42.39 & \\
\hline
\end{tabular}

${ }^{a}$ From Barger et al. (2003): s, less secure redshift identification based primarily on a single emission line and the continuum shape; $\mathrm{m}$, complex or multiple structure or possible contamination of the photometry by a nearby brighter object.
Table 6. Previously Known Type 2 AGN, CDF-S.

\begin{tabular}{|c|c|c|c|c|}
\hline $\mathrm{A} 03$ & S04 & redshift & $\begin{array}{c}\log L_{\mathrm{x}}(2-8 \mathrm{keV}) \\
\mathrm{erg} / \mathrm{s}\end{array}$ & Notes $^{a}$ \\
\hline 44 & 66 & 0.574 & 43.31 & \\
\hline 48 & 267 & 0.720 & 43.07 & $\mathrm{QF}=1$ \\
\hline 60 & 155 & 0.545 & 42.11 & \\
\hline 68 & 62 & 2.810 & 44.48 & \\
\hline 80 & 535 & 0.575 & 42.20 & \\
\hline 84 & 149 & 1.033 & 42.81 & $\mathrm{QF}=1$ \\
\hline 86 & 57 & 2.562 & 44.28 & \\
\hline 88 & $56 a$ & 0.605 & 43.41 & \\
\hline 90 & 600 & 1.327 & 42.86 & \\
\hline 91 & 266 & 0.735 & 42.43 & \\
\hline 96 & 531 & 1.544 & 43.19 & \\
\hline 118 & $51 \mathrm{a}$ & 1.097 & 44.06 & \\
\hline 123 & 153 & 1.536 & 43.97 & \\
\hline 126 & 50 & 0.670 & 42.61 & $\mathrm{QF}=1$ \\
\hline 131 & 253 & 0.481 & 42.53 & $\mathrm{QF}=1$ \\
\hline 134 & 151 & 0.604 & 43.02 & \\
\hline 137 & $612 b$ & 0.736 & 42.55 & \\
\hline 146 & 188 & 0.734 & 42.14 & \\
\hline 160 & $606 a$ & 1.037 & 42.89 & $\mathrm{QF}=1$ \\
\hline 161 & 47 & 0.733 & 43.08 & \\
\hline 162 & 260 & 1.043 & 42.83 & \\
\hline 164 & 150 & 1.090 & 43.34 & \\
\hline 166 & 45 & 2.291 & 44.20 & $\mathrm{QF}=1$ \\
\hline 176 & 43 & 0.734 & 42.90 & \\
\hline 179 & 41 & 0.668 & 43.31 & \\
\hline 188 & 202 & 3.700 & 44.47 & \\
\hline 212 & 512 & 0.668 & 42.08 & \\
\hline 220 & 190 & 0.735 & 43.07 & \\
\hline 245 & 27 & 3.064 & 44.64 & \\
\hline 247 & 25 & 0.625 & 43.15 & $\mathrm{QF}=0.5$ \\
\hline 249 & $611 \mathrm{a}$ & 0.979 & 42.74 & $\mathrm{QF}=1$ \\
\hline 263 & $20 a$ & 1.016 & 43.40 & \\
\hline 269 & 170 & 0.664 & 42.38 & \\
\hline 271 & 252 & 1.178 & 43.29 & \\
\hline 276 & 184 & 0.667 & 42.54 & \\
\hline
\end{tabular}

${ }^{a}$ From Szokoly et al. (2004): QF $=1$, spectrum cannot be identified securely, typically only a single narrow line present; $\mathrm{QF}=0.5$, only a hint of some spectral feature.

Szokoly et al. 2004). We also note that with a lower X-ray power cut of $L_{2-8}>5 \times 10^{43} \mathrm{erg} / \mathrm{s}$ the number of QSO 2 increases to 13 .

Figure 5 shows the $\mathrm{X}$-ray power distribution for our new type 2 AGN candidates (dashed line), previously known type 2 AGN (solid line), and the combined sample (dotted line). It is interesting to note how the distributions are very different, with the already known type 2 AGN peaking around $L_{\mathrm{x}} \sim 10^{43} \mathrm{erg} / \mathrm{s}$ and declining for luminosities above $\sim 3 \times$ $10^{43} \mathrm{erg} / \mathrm{s}$, while our new candidates are rising in this range and 


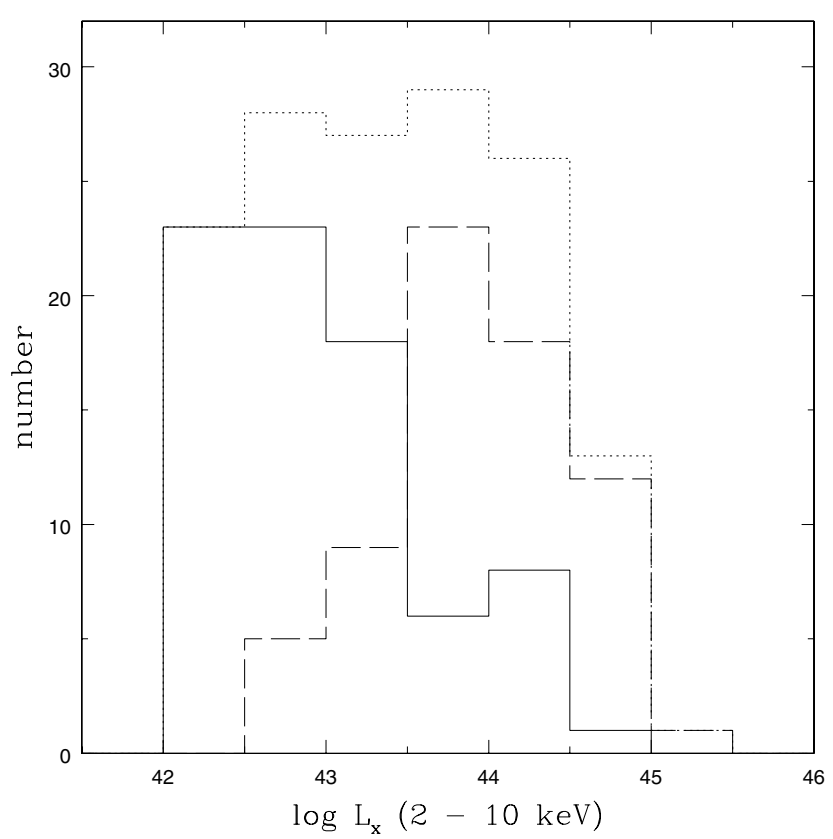

Fig. 5. The X-ray power distribution for our new type 2 AGN candidates (dashed line), previously known type 2 AGN (solid line), and the sum of the two populations (dotted line). QSO 2 are defined, somewhat arbitrarily, as having $L_{2-10 \mathrm{kev}}>10^{44} \mathrm{erg} / \mathrm{s}$.

peak around $L_{\mathrm{x}} \sim 10^{44} \mathrm{erg} / \mathrm{s}$. To be more quantitative, while only $\sim 1 / 5$ of already known type 2 AGN have $\log L_{\mathrm{x}}>43.5$, $\sim 3 / 4$ of our candidates are above this value. This difference is easily explained by our use of the X-ray-to-optical flux ratios to estimate X-ray powers and by the fact that our candidates are on average $\sim 3$ mag fainter than previously known sources. Our method is then filling a gap in the luminosity distribution, which becomes almost constant in the range $10^{42} \lesssim L_{\mathrm{x}} \lesssim 3 \times 10^{44} \mathrm{erg} / \mathrm{s}$. This also explains the fact that the number of QSO 2 candidates we find is $\geq 3$ times larger than the previously known ones.

Conversely the fluxes of the already known QSO 2 reach $f(2-8 \mathrm{keV}) \sim 3 \times 10^{-15} \mathrm{erg} \mathrm{cm}^{-2} \mathrm{~s}^{-1}$, while our new QSO 2 candidates go down to the fainter limit of $f(2-8 \mathrm{keV}) \sim$ $4 \times 10^{-16} \mathrm{erg} \mathrm{cm}^{-2} \mathrm{~s}^{-1}$. This is explained by the much fainter optical magnitudes we are probing with our method which, for a given $\mathrm{X}$-ray-to-optical flux ratio, translate into fainter $\mathrm{X}$-ray fluxes as well.

By going fainter we are also probing the type 2 AGN population at higher redshifts. While our estimated redshift is $\left\langle z_{\text {est }}\right\rangle \sim 2.9$ (median 2.6), previously known type 2 AGN have $\langle z\rangle \sim 1.1$ (median 0.9). For QSO 2 we find $\left\langle z_{\text {est }}\right\rangle \sim 3.7$ (median 3.5), as compared to $\langle z\rangle \sim 2.3$ (median 2.6).

\subsection{Total number of type 2 AGN}

We find a total of 147 type 2 AGN in the GOODS ACS area, 79 of which were already known. This corresponds to $27_{-2}^{+3} \%$ of the 546 sources in the Alexander et al. (2003) catalogue ( $14_{-2}^{+2} \%$ including the previously known sources only). As regards QSO 2, the total number is 40, only 9 of which were previously known. Our method has therefore more than quadrupled the number of such sources in the GOODS ACS fields. These represent $7_{-1}^{+1} \%$ of all (546) X-ray sources (the previously known QSO 2 make up $2_{-1}^{+1} \%$ ).

Note that all of these sources satisfy the commonly adopted definition of absorbed source discussed above. In fact, Fig. 12 of Szokoly et al. (2004) shows that $H R \geq-0.2$ corresponds to $N_{\mathrm{H}}>10^{22} \mathrm{~cm}^{-2}$, for an intrinsic $\alpha_{\mathrm{x}}=1$, for $z \gtrsim 0.4$. The lowest redshift for previously known type $2 \mathrm{AGN}$ is 0.4 , while the lowest estimated redshift for our type 2 candidates is 0.9 . The typical $H R$ and redshifts for our sources imply that we are dealing with $N_{\mathrm{H}} \approx 10^{23}$ and $\approx 3 \times 10^{23} \mathrm{~cm}^{-2}$ for the known and candidate sources respectively.

$\mathrm{X}$-ray obscuration can be also present in a small fraction of broad-lined AGN. Perola et al. (2004, and references therein) find this to be the case in $\sim 10 \%$ of their sources, with estimated $N_{\mathrm{H}} \gtrsim 10^{22} \mathrm{~cm}^{-2}$. As discussed in the previous paragraph, these sources are then likely to be at relatively low redshift. Therefore, contamination by type 1 AGN in our sample is expected to be negligible.

\subsection{The surface density of QSO 2}

The detection of faint type 2 AGN candidates and the careful assessment of the previously known such sources in the two GOODS fields allow us to put strong constraints on the surface density of type 2 sources, and in particular on that of QSO 2. We use a value for the Viz-band coverage of $365 \operatorname{arcmin}^{2} \simeq$ $0.1014 \mathrm{deg}^{2}$ (Giavalisco et al. 2004a).

Due to the variable sensitivity of the Chandra ACIS-I detector across the field of view, the area in which fainter X-ray sources could be detected is smaller than that for brighter sources. In other words, the sky coverage is not uniform at all fluxes. Figure 5 of Giacconi et al. (2002) and Fig. 19 of Alexander et al. (2003), however, show that the effect is strong only at relatively small fluxes. For example, for both CDF-S and HDF-N the effective area decreases in the hard band, for which this effect is strongest, by $\gtrsim 25 \%$ only for $f(2-8 / 10 \mathrm{keV}) \lesssim 10^{-15} \mathrm{erg} \mathrm{cm}^{-2} \mathrm{~s}^{-1}$.

To better quantify the magnitude of this correction we evaluated the sky coverage for the combined GOODS fields by simply scaling the two sky coverages so that the maximum area for each field was equal to half the total GOODS area, summing then up the two areas. This works only as a first approximation and slightly overestimates the correction since the GOODS ACS fields are at the centre of the Chandra fields and therefore deeper than average in the X-ray band. In other words, the sky coverage should be re-computed as its shape would change, with a larger area accessible at fainter fluxes and therefore a smaller correction than we estimate. In any case, under our assumptions we find that the correction is within the $1 \sigma$ Poisson range, and therefore within the statistical uncertainties, for $f(0.5-8 \mathrm{keV}) \gtrsim 10^{-15} \mathrm{erg} \mathrm{cm}^{-2} \mathrm{~s}^{-1}$. Our conservative approach is then to not to take the sky coverage into account and limit ourselves to X-ray fluxes $\geq 10^{-15} \mathrm{erg} \mathrm{cm}^{-2} \mathrm{~s}^{-1}$. This means that our values are actually lower limits, although the real surface densities should not be more than $25 \%$ larger. 
Table 7. QSO 2 surface density.

\begin{tabular}{|c|c|c|c|c|c|c|}
\hline \multirow{2}{*}{$\begin{array}{l}f(0.5-8 \mathrm{keV}) \\
\mathrm{erg} \mathrm{cm}^{-2} \mathrm{~s}^{-1}\end{array}$} & \multicolumn{3}{|c|}{$N$} & \multicolumn{3}{|c|}{$N\left(>f_{\mathrm{x}}\right)$} \\
\hline & Known & Candidate & Total & Known & $\begin{array}{c}\text { Candidate } \\
\mathrm{deg}^{-2}\end{array}$ & Total \\
\hline $1 \times 10^{-15}$ & 9 & 25 & 34 & $89_{-29}^{+40}$ & $247_{-49}^{+60}$ & $335_{-57}^{+68}$ \\
\hline $2 \times 10^{-15}$ & 9 & 14 & 23 & $89_{-29}^{+40}$ & $138_{-36}^{+48}$ & $227_{-47}^{+58}$ \\
\hline $5 \times 10^{-15}$ & 7 & 3 & 10 & $69_{-25}^{+37}$ & $30_{-16}^{+29}$ & $99_{-31}^{+42}$ \\
\hline $1 \times 10^{-14}$ & 3 & 1 & 4 & $30_{-16}^{+29}$ & $10_{-8}^{+23}$ & $39_{-19}^{+31}$ \\
\hline
\end{tabular}

Table 7 gives the QSO 2 surface density for four flux limits, splitting the contribution into known and candidate sources for the full $0.5-8 \mathrm{keV}$ band. (The counts in the hard band are not very different, since we are dealing with absorbed sources, with the total numbers changing to 33 (from 34) and 8 (from 10) for the first and third flux limit respectively.)

These numbers can be compared with recent estimates and predictions. Perola et al. (2004) find a surface density of highly obscured QSO, which they define as having $L_{2-10}>$ $10^{44} \mathrm{erg} / \mathrm{s}$ and $N_{\mathrm{H}}>10^{22} \mathrm{~cm}^{-2}, \sim 48 \mathrm{deg}^{-2}$ for $f(2-10 \mathrm{keV})>$ $10^{-14} \mathrm{erg} \mathrm{cm}^{-2} \mathrm{~s}^{-1}$, consistent with our value. At lower fluxes the situation is different. For example, for $f(0.5-7 \mathrm{keV})>$ $5 \times 10^{-15} \mathrm{erg} \mathrm{cm}^{-2} \mathrm{~s}^{-1}$ Gandhi et al. (2004) quote an estimated value $>3 \mathrm{deg}^{-2}$ and possibly higher than $10 \mathrm{deg}^{-2}$ and a predicted value from their model of $19 \mathrm{deg}^{-2}$. We find a density $\sim 100 \mathrm{deg}^{-2}$, that is a factor $\gtrsim 10$ larger than their estimate and $\sim 5$ times larger than their prediction. Already known sources make up $\sim 70 \%$ of our total value, so this high density is clearly not attributable only to our new candidates. We do note that 5 out of the 7 previously known QSO 2 come from the paper by Szokoly et al. (2004), which appeared only recently and could not be accounted for by Gandhi et al. (2004). For the same flux limit and $L_{2-10}>3 \times 10^{44} \mathrm{erg} / \mathrm{s}$ these authors predict a surface density $\sim 3 \mathrm{deg}^{-2}$, while we find 5 sources ( 3 of them already known), which implies a density $49_{-21}^{+33} \mathrm{deg}^{-2}$. The decrease in space density at high luminosity is therefore much less than predicted by their model, namely only a factor $\sim 2$ instead of $\sim 6$.

At even fainter fluxes, Gandhi et al. (2004) quote a value from their model of $35 \mathrm{deg}^{-2}$ above $f(0.5-7 \mathrm{keV}) \sim 2 \times$ $10^{-15} \mathrm{erg} \mathrm{cm}^{-2} \mathrm{~s}^{-1}$, while we find a density $\sim 230 \mathrm{deg}^{-2}$, that is a factor $\sim 6$ larger than their prediction.

The population synthesis model of Gilli et al. (2001, and private communication) predicts 16 obscured QSO with intrinsic luminosity $L_{2-10}>3 \times 10^{44} \mathrm{erg} / \mathrm{s}, N_{\mathrm{H}}>10^{22} \mathrm{~cm}^{-2}$, and $z>3$ in the 1 Ms CDF-S. We find 2 known plus 7 candidate sources above these redshift and power limits with $H R \geq-0.2$. According to Fig. 12 of Szokoly et al. (2004) at $z \gtrsim 3$ an $N_{\mathrm{H}}=10^{22} \mathrm{~cm}^{-2}$ corresponds to $H R \sim-0.6$ for an intrinsic $\alpha_{\mathrm{x}}=1$, which means that our definition is more restrictive. Considering also that the southern GOODS ACS area is $\sim 60 \%$ smaller than the CDF-S area, there are strong indications that our number might be a very solid lower limit to such sources and that therefore our findings are not inconsistent with the numbers predicted by Gilli et al. (2001).
As mentioned in Sect. 5, the number of our QSO 2 candidates depends on the $\log L_{2-10}-\log f(2-10 \mathrm{keV}) / f(R)$ correlation of Fiore et al. (2003), which has an rms of $\sim 0.5 \mathrm{dex}$ in X-ray power. However, even if we consider the worst case scenario in which all our estimated X-ray powers are too large by 0.5 dex the number of QSO 2 candidates for the four flux limits in Table 7 are 13, 8, 2, and 1 respectively. In other words, our total densities decrease, at worst, by $\sim 25-35 \%$ at the lowest fluxes, and are basically unchanged at larger fluxes.

The resolved fraction of the X-ray background due to QSO 2 down to $f(2-8 \mathrm{keV})=10^{-15} \mathrm{erg} \mathrm{cm}^{-2} \mathrm{~s}^{-1}$ is estimated to be $10_{-2}^{+2} \%$. We have used here the value of the total X-ray background measured by UHURU and HEAO-1 (Marshall et al. 1980) and the uncertainties reflect the rms of the Fiore et al. (2003) correlation (the Poissonian error is even smaller). Note that given the relatively small area of the GOODS fields we are missing the bright end of the number counts. In fact, only four of our sources have $f(2-8 \mathrm{keV})>10^{-14} \mathrm{erg} \mathrm{cm}^{-2} \mathrm{~s}^{-1}$ and these reach only $2.6 \times$ $10^{-14} \mathrm{erg} \mathrm{cm}^{-2} \mathrm{~s}^{-1}$. This fact, the discussion above and the points detailed in the next section, all mean that our value has to be regarded as a robust lower limit.

\subsection{Caveats and comments}

In this paper we have employed a statistical method to identify very faint type 2 AGN. We had also to rely on an empirical technique to estimate the X-ray powers, which were needed to make sure that the sources we are dealing with have AGN-like outputs. As such, we can only provide a list of candidates and not firm classifications. This was obviously expected, as the great majority of our candidates are so faint that even the largest telescopes presently available would require extremely long exposures to secure a decent spectrum.

However, we believe that our method is robust, as shown by the various checks we have performed. Importantly, we have been very conservative in our estimates of the number of type 2 AGN candidates and the surface densities we have estimated need to be considered lower limits (modulo what discussed in the previous paragraph), for the following four reasons: 1. our selection criterion for absorbed sources $(H R \geq-0.2)$ will mistakenly discard some high- $z$ type 2 sources (Sect. 4.1); 2. the same criterion is also more restrictive than the commonly used one based on $N_{\mathrm{H}}$ (Sect. 6.2); 3. the $\mathrm{X}$-ray powers for previously known sources have not been 
corrected for absorption, which means more sources could be above the QSO 2 limit (Sect. 4.3); and finally, 4. sky coverage effects, once properly taken into account, will reduce the available area at smaller X-ray fluxes and therefore increase the source surface density (Sect. 6.3).

\subsection{Next steps enabled with VO tools}

As noted above, a remaining source of uncertainty with the newly discovered sample is the reliance on an empirical technique in the determination of the X-ray power of the objects.

In Sect. 5.1 we noted how the 4 colour ACS photometric data have been used to estimate redshifts for a number of objects in a restricted range. Future work will enable the use of the 4 band ACS plus IR (VLT/ISAAC and Spitzer) photometry to determine photometric redshifts over the full GOODS field. VO technologies are to be employed to facilitate this work. Upgrades to the AVO capability will include the provision of a "redshift-determination" service, which will automate the generation of point spread function matched multi-band input photometric catalogues via the use of AstroGrid's (Walton et al. 2003) ACE (Astronomical Catalogue Extractor) service (a Web service providing access to the SExtractor application, Bertin \& Arnouts 1996), and feed these into a range of photometric redshift determination techniques (e.g., Bayesian prior method, Bpz, Benítez 2000; the neural network technique, ANNz, Collister \& Lahav 2004; and Hyperz, Bolzonella et al. 2000).

It is necessary to include the IR data in order to obtain reliable photometric redshifts, and with the additional photometric bands, the effects of possible dust extinction will also be identifiable (Rowan-Robinson 2003).

\section{Conclusions}

We have used Virtual Observatory tools to identify obscured AGN much fainter than previously known ones, going beyond what is currently possible using the "classical" approach of classifying sources by taking spectra of them even using the largest telescopes available today. The fact that we have obtained scientifically useful and cutting-edge results is proof that VO tools have evolved beyond the demonstration level to become respectable research tools. The VO is already enabling astronomers to reach into new areas of parameter space with relatively little effort.

Our main results can be summarized as follows:

1. By employing publicly available, high-quality X-ray and optical data we have discovered 68 type 2 AGN candidates in the two GOODS fields. Their X-ray powers have been estimated by using a previously known correlation between X-ray luminosity and X-ray-to-optical flux ratio. Thirty-one of our candidates have high luminosity $\left(L_{2-10 \mathrm{keV}}>10^{44} \mathrm{erg} / \mathrm{s}\right)$ and therefore qualify as QSO 2, that is optically obscured quasars. Based on their derived $\mathrm{X}$-ray powers, our candidates are likely to be at relatively high redshifts, $z \sim 3$, with the QSO 2 at $z \sim 4$.
2. We have tested our method and results extensively and find them to be very robust. In particular: a) our approach recovers most ( 97\%) of the previously known type 2 AGN in the GOODS fields; b) the X-ray power estimates agree very well with those derived from spectroscopic redshifts for the non-type $1 \mathrm{AGN}$ in the GOODS fields; c) the redshifts derived from our estimated powers are consistent with those measured for previously known non-type 1 AGN; d) the spectrum of our brightest northern candidate, recently made public as part of the Team Keck Treasury Redshift Survey, confirms our type 2 classification as it is basically featureless, with a measured redshift extremely close to our estimate.

3. By going $\sim 3$ mag fainter than previously known type 2 AGN we are sampling a region of redshift power space so far unreachable with classical methods. Our method brings to 40 the number of QSO 2 in the GOODS fields, an improvement of a factor $\sim 4$ when compared to the 9 such sources previously known. The relatively large QSO 2 number density we derive $\left(\gtrsim 330 \mathrm{deg}^{-2}\right.$ for $f(0.5-8 \mathrm{keV}) \geq 10^{-15} \mathrm{erg} \mathrm{cm}^{-2} \mathrm{~s}^{-1}, \sim 30 \%$ of which is made up of already known sources) suggests that its value at faint flux limits has been underestimated.

For the handful of our candidates with $R \lesssim 26$ spectroscopy with a class $8-10 \mathrm{~m}$ telescope is still within reach, albeit with relatively long $(\$ 10 \mathrm{~h})$ exposure times. We plan to follow these up and confirm (or refute) their classification in the near future.

In closing we note that this paper represents the first significant published science result that has been fully enabled via end-to-end use of VO tools and systems.

VO initiatives are in their early stages. Significant progress is being made both by the AVO and other national VO projects. Construction of a truly pervasive system is beginning to provide the end user access to a powerful combination of data access, applications embedded within user workflows, running over high speed networks on powerful compute resources. Early use, exploiting a prototype system in the USA, constructed by the US-VO project ${ }^{14}$ to simplify the cross matching of Two Micron All Sky Survey and Sloan Digital Sky Survey source catalogues has enabled Berriman et al. (2003) to discover a small number of hitherto undiscovered Brown Dwarf candidates. Their work demonstrated possibilities, but did not alter the scientific understanding of that particular problem.

In this paper we have shown how, even with the AVO's early prototype, enough capability and access to data is available to make the scientific mining of the, in this case GOODS, data significantly easier for the "general" astronomer. This is because gains in interoperability simplify the acquisition of the data, and enable quick interoperation of a number of common, but computationally intensive, tasks such as cross matching and multi layer visualisation of both image and catalogue data.

With the rapid evolution of the VO, science discovery will be routinely performed using VO techniques, an early example of which is described here.

\footnotetext{
${ }^{14}$ http://www.us-vo.org
} 
The AVO prototype used in this paper is freely available for download $^{15}$.

Acknowledgements. We thank Fabrizio Fiore for providing the data on which the X-ray power - X-ray-to-optical flux ratio correlation was based, Roberto Gilli, Vincenzo Mainieri, and Paolo Tozzi for useful discussions, Sebastien Derriere for assistance with the false match rate estimates, and the AVO team for their superb work, without which this paper would have not been possible. Finally, a sincere "thank you" to the many people who have produced the data on which this paper is based, particularly the GOODS, CDF-S, and Penn State Teams. This research has made use of the CDS Vizier catalogue tool, SIMBAD and Aladin sky atlas services.

\section{Appendix A: False match estimate}

We estimated the number of false matches using the formalism described in Derriere (2001). The expected total number of matches $D$, for $N_{1}$ objects with positional uncertainty $\sigma$ against a uniformly distributed set of objects with density $\lambda$ is:

$$
\begin{aligned}
& D\left(d, N_{1}, \lambda, \sigma, N\right)= \\
& N_{1} \Phi(d, \lambda)+N \Phi(d, \lambda)\left[\frac{1+2 \pi \lambda \sigma^{2}}{2 \pi \lambda \sigma^{2}} \exp \left(-\frac{d^{2}}{2 \sigma^{2}}\right)-1\right]
\end{aligned}
$$

where $d$ is the distance between matches, $\Phi(d, \lambda)$ is the 2-d Poisson density function ${ }^{16}$ and $N$ is the number of objects with a true counterpart. $D$ may also be expressed a sum of three components, $\alpha^{17}$ the number of true matches, $\beta^{18}$ the objects with a true partner but not incorrectly assigned and $\psi^{19}$ the number of objects that do not have a counterpart but have been matched.

Using our known values of $N_{1}$ : the number of absorbed sources (203), $\lambda$ : the optical source density of the GOODS catalogues $\left(0.0469 \operatorname{arcsec}^{-2}\right)$, and $\sigma$ : the median positional error of the X-ray positions of the absorbed sources $\left(0.31^{\prime \prime}\right)$ we vary $N$ to perform a maximum likelihood fit of the expected distribution of matches $D$ to the observed histogram of distances between closest match optical and X-ray sources out to the initial 3.5" threshold radius. The best fit occurs for $N=160$. Figure A. 1 shows the histogram of distances between closest match optical and X-ray sources overlaid with the best fitting model.

Integrating $D$ and $\alpha$ out to $1.25^{\prime \prime}$ we find that $92 \%$ of the matches out to this radius are expected to be true according to the model, making our false match fraction $8 \%$. Note that this approach neglects clustering, which would slightly increase this value.

We also estimated the number of false matches by comparing the number of X-ray to optical matches we find using the correct coordinates, to the number of matches we find when a shift (of $20^{\prime \prime}$ much larger than the typical match radius and the

\footnotetext{
15 http://www. euro-vo.org/twiki/bin/view/Avo/ SwgDownload

$16 \Phi(d, \lambda)=2 \pi \lambda d \exp \left(-\pi \lambda d^{2}\right)$.

$17 \alpha=\frac{N}{2 \pi \lambda \sigma^{2}} \Phi(d, \lambda) \exp \left(-\frac{d^{2}}{2 \sigma^{2}}\right)$.

$18 \beta=N \Phi(d, \lambda) \exp \left(-\frac{d^{2}}{2 \sigma^{2}}\right)$.

${ }_{19} \psi=\left(N_{1}-N\right) \Phi(d, \lambda)$.
}

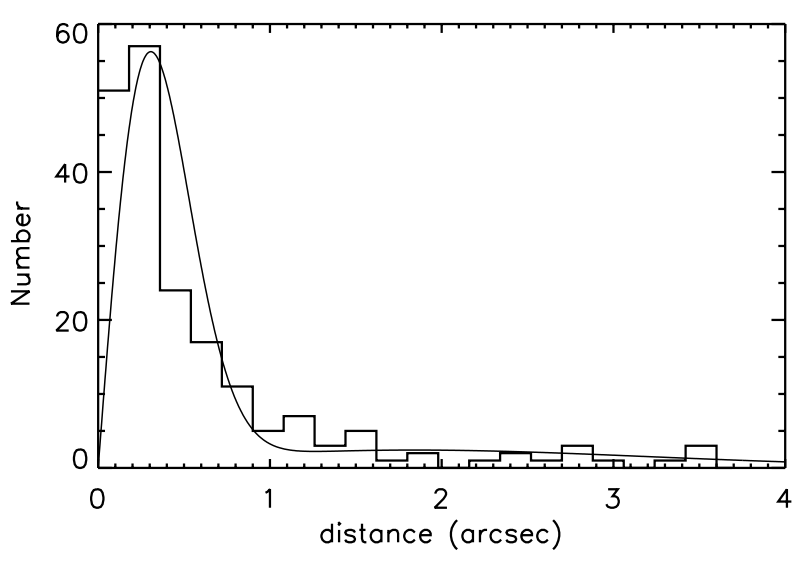

Fig. A.1. The histogram of distances between closest match optical and X-ray sources overlaid with the best fitting model.

spacing between optical sources) is applied to the X-ray coordinates. Within $1.25^{\prime \prime}$ we find 188 matches and 38 matches when the coordinates are shifted. Our selection of real matches however, also included the distance/error criterion which means that a number of matching sources within $1.25^{\prime \prime}$ of the X-ray source were discarded. This criterion should also be taken into consideration when estimating the rate of false matches. We estimate this effect on the false match rate by calculating the rate of optical sources within $1.25^{\prime \prime}$ of the real X-ray position which have distance/error $>1$ (10), and then subtract this from the rate of false matches we find in the offset cross-match. Therefore the false match fraction using this method should be $(38-10) / 188=15 \%$.

The fraction of false matches should then be in the range $8-15 \%$.

\section{References}

Alexander, D. M., Bauer, F. E., Brandt, W. N., et al. 2003, AJ, 126, 539

Barger, A. J., Cowie, L. L., Capak, P., et al. 2003, AJ, 126, 632

Beckwith, S. V. W., et al. 2004, in preparation

Benítez, N. 2000, ApJ, 536, 571

Berriman, B., Kirkpatrick, D., Hanisch, R., Szalay, A., \& Williams, R. 2003, Large Telescopes and Virtual Observatory: Visions for the future, 25th Meet. of the IAU, Joint Discussion 8, 17 July, Sydney, Australia, in press

Bertin, E., \& Arnouts, S. 1996, A\&A, 117, 393

Bolzonella, M., Miralles, J.-M., \& Pello, R. 2000, A\&A, 363, 476

Bonnarel, F., Fernique, P., Bienaymé, O., et al. 2000, A\&AS, 143, 33

Cohen, J. G. 2003, ApJ, 598, 288

Collister, A. A., \& Lahav, O. 2004, PASP, 116, 345

Comastri, A., Fiore, F., Vignali, C., et al. 2001, MNRAS, 327, 781

Cristiani, S., Alexander, D. M., Bauer, F., et al. 2004, ApJ, 600, L119

Derriere, S. 2001, Ph.D. Thesis (Annexe B)

Fiore, F., Brusa, M., Cocchia, F., et al. 2003, A\&A, 409, 79

Forman, W., Jones, C., \& Tucker, W. 1994, ApJ, 429, 77

Gandhi, P., Crawford, C. S., Fabian, A. C., \& Johnstone, R. M. 2004, MNRAS, 348, 529

Gehrels, N. 1986, ApJ, 303, 336

Giacconi, R., Zirm, A., Wang, J. X., et al. 2002, ApJS, 139, 369

Giavalisco, M., Ferguson, H. C., Koekemoer, A. M., et al. 2004a, ApJ, 600, L93 
Giavalisco, M., \& the GOODS Team 2004b, in preparation Gilli, R., Salvati, M., \& Hasinger, G. 2001, A\&A, 366, 407 Isobe, T., Feigelson, E. D., Akritas, M. G., \& Babu, G. J. 1990, ApJ, 364, 104

Jaffe, W., Meisenheimer, K., Röttgering, H. J. A., et al. 2004, Nature, 429,47

Le Fèvre, O., Vettolani, P., Paltani, S., et al. 2004, A\&A, submitted

Maccacaro, T., Gioia, I. M., Wolter, A., Zamorani, G., \& Stocke, J. T. 1988, ApJ, 326, 680

Marshall, F. E., Boldt, E. A., Holt, S. S., et al. 1980, ApJ, 235, 4

Mobasher, B., Idzi, R., Benítez, N., et al. 2004, ApJ, 600, L167

Moran, E. C., Filippenko, A. V., \& Chornock, R. 2002, ApJ, 579, L71

Norman, C., Hasinger, G., Giacconi, R., et al. 2002, ApJ, 571, 218
Ochsenbein, F., Bauer, P., \& Marcout, J. 2000, A\&AS, 143, 23

Perola, G. C., Puccetti, S., Fiore, F., et al. 2004, A\&A, 421, 491

Rowan-Robinson, M. 2003, MNRAS, 345, 819

Shapley, A., Fabbiano, G., \& Eskridge, P. B. 2001, ApJS, 137, 139

Szokoly, G. P., Bergeron, J., Hasinger, G., et al. 2004, ApJS, in press [arXiv:astro-ph/0312324]

Urry, C. M., \& Padovani, P. 1995, PASP, 107, 803

Vanzella, E., et al. 2004, in preparation

Walton, N. A., Lawrence, A., \& Linde, A. E. 2003, ASASS, 12, 25

Wirth, G. D., Willmer, C. N. A., Amico, P., et al. 2004, AJ, 127, 3121

Zombeck, M. V. 1990, Handbook of Space Astronomy and Astrophysics (Cambridge University Press) 